le portiQue $\begin{array}{ll}\text { Le Portique } \\ \text { Revue de philosophie et de sciences humaines }\end{array}$

33 | 2014

Straub !

\title{
Après O Somma Luce et Corneille
}

\author{
Jean-Marie Straub
}

\section{(2) OpenEdition}

\section{Journals}

\section{Electronic version}

URL: http://journals.openedition.org/leportique/2760

DOI: 10.4000/leportique.2760

ISSN: $1777-5280$

\section{Publisher}

Association "Les Amis du Portique"

Printed version

Date of publication: 1 May 2014

ISSN: 1283-8594

\section{Electronic reference}

Jean-Marie Straub, "Après O Somma Luce et Corneille", Le Portique [Online], 33 | 2014, document 3, Online since 05 February 2016, connection on 12 April 2021. URL: http://journals.openedition.org/ leportique/2760 ; DOI: https://doi.org/10.4000/leportique.2760

This text was automatically generated on 12 April 2021.

Tous droits réservés 


\title{
Après O Somma Luce et Corneille
}

\author{
Jean-Marie Straub
}

1 Un étudiant - Je voudrais savoir quelle différence vous faites entre un film en pellicule et un film numérisé.

2 Jean-Marie Straub - La pellicule est beaucoup plus définie et a plus d'informations. Le numérique est plus pictural.

3 L'étudiant - Ça nous intéresse en tant qu'étudiants aux Beaux-arts, par exemple.

4 J.-M. S. - Vous feriez mieux de me dire ce que vous avez vu, vous.

5 L'étudiant - Ce que j'ai vu, moi, de vous, ou de...?

6 J.-M. S. - Comme différence.

7 L'étudiant - J'ai trouvé que c'était vachement plus froid qu'en pellicule. C'est plus chaud avec les pellicules en tout cas. On a peut-être des imperfections comme ça, mais les couleurs sont plus chaudes.

8 J.-M. S. - En numérique?

9 L'étudiant - Non, en pellicule, en $35 \mathrm{~mm}$, c'est vachement plus chaleureux comme image.

10 J.-M. S. - Pourquoi pas ? Je suis incapable de vous répondre parce que... Il y a un choix qui dépend du film qu'on veut faire, c'est tout. Une matière qui est différente selon les cas. Naturellement que le progrès numérique n'est pas un progrès absolu, loin de là. Il est associé à une régression énorme, mais comme tout progrès, ce n'est pas seulement une régression.

11 Bernard Muscat (Ciné Art) - Dans le film de Pedro Costa, on voit un banc de montage 35 $\mathrm{mm}$. Est-ce que c'est différent de faire du montage avec de la pellicule $35 \mathrm{~mm}$ ? Est-ce que c'est plus artisanal? Je me demande si ce n'est pas au moment du montage qu'il y a une grande différence...

12 J.-M. S. - En 35, c'est une affaire matérielle. On a quelque chose qu'on touche, c'est des longueurs, avec des perforations, etc. En numérique, on fait tic-tic... 
13 L'étudiant - Oui, je connais un petit peu. On fait de la photo aux Beaux-Arts, et de la photo argentique aussi... Les différences entre photo argentique et photo numérique ne sont pas forcément les mêmes qu'entre cinéma en pellicule et cinéma numérique. Un spectateur - Un et demi. Je voudrais savoir si vous avez choisi le morceau de Dante au hasard ou si c'est quelque chose qui vous touche personnellement. Est-on autorisé à penser que lorsque l'on vous fait des compliments, vous n'aimez pas qu'on vous en fasse ? Chaque fois, vous bougonnez ! Cela a-t-il un rapport avec les vers de Dante ? La distance entre la vision qu'il a lui et celle que vous avez vous? Tout ce que vous pouvez faire, c'est en rendre une toute petite partie, et ça, ça vous agace, ça vous frustre... Vous n'êtes pas le seul ; il y a beaucoup d'artistes qui... Proust, par exemple, quand il parle de la mort de Bergotte, il dit aussi : j'aurais dû m'y prendre autrement, maintenant c'est trop tard, qu'est-ce que j'ai fait ? Ceux qui vous disent : « non, c'est pas mal, c'est bien », eh bien, ça vous agace. Est-ce qu'il y a un lien entre les deux ? Est-ce qu'on peut penser qu'il y a un lien entre les deux? J.-M. S. - Rien n'est tout à fait au hasard.

Un spectateur - Mais, est-ce que vous ressentez ça comme ça ? Que ce que vous arrivez à rendre est insuffisant par rapport à ce que vous voudriez rendre... ?

J.-M. S. - Non, ça c'est du romantisme. On travaille, on essaie d'obtenir ce qu'on a envie d'obtenir. C'est tout. Les histoires d'insatisfaction des artistes, c'est de la pataphysique.

Un spectateur - Cet extrait, vous l'avez choisi, quand même... Et ça parle en grande partie de ça...

J.-M. S. - Écoutez, vous serez là demain, par hasard?

Un spectateur - Pour La mort d'Empédocle? Oui.

J.-M. S. - Là, il y a un hymne à la lumière qui est le côté Hölderlin de la question. Si je n'avais pas fait ça avant, je ne me serai pas intéressé à ce... C'est exactement la fin de tout le Dante, c'est la fin du « Paradis », qui est le troisième chant de la Divine Comédie. C'est tout. Évidemment que si je l'ai choisi, c'est que ça m'intéressait plus que d'autres passages. C'est tout. Qu'il y ait là-dedans ce que vous dites, je n'en sais rien. Je pense que ce n'est pas à moi à le dire.

Un spectateur - Tout ce que je voulais savoir, c'est, si quelqu'un écrivait ce que je viens de dire, est-ce que vous vous fâcheriez?

J.-M. S. - Non.

Un spectateur - Non ? Voilà.

J.-M. S. - Faire ce travail, c'est un luxe, un privilège qu'on a par rapport à $90 \%$ des gens qui, aujourd'hui, font un travail qui ne les intéresse pas. Donc, on ne peut pas se permettre de le faire d'une manière qui vous frustre ou qui ne vous satisfasse pas ; ça n'aurait pas de sens. Sinon, il faut changer de métier. J'admets qu'il y a quelque chose dans ce que vous dites, mais je n'aime pas beaucoup m'y complaire. On y va? 